areas: patient population, outcomes, and prescribing and administration patterns.

Methods A retrospective review of electronic prescriptions and clinical records was undertaken in April 2017. All patients who were prescribed AMs using the hospital electronic AP protocol from 1 st July to 31 st Sept 2016 were identified. Demographic, clinical and outcome data were collected for all identified patients $(n=118)$. Prescribing and administration patterns were reviewed for a representative subsample $(n=50)$.

Results 118 patients were prescribed AMs (mean age: 81.2 \pm 10.3 years, 63 men). $38(32.3 \%)$ had malignant disease and $49(41.5 \%)$ had inpatient palliative team involvement. $66 \%$ $(n=78)$ died in hospital, representing $49.7 \%$ of all inpatient deaths in that 3 month period. Median time from AM prescription to inpatient death was 1 day (IQR: 0-3). 34\% $(n=40)$ of those prescribed AMs as an inpatient were discharged. Of these, $33(82.5 \%)$ had died within 6 months, a median of 8 days (IQR: 4-26) after discharge; AMs were omitted from the discharge prescription in 30\%. For the 50patient subsample, AMs were administered in 54\% of cases, usually within a few hours of death.

Conclusions There appeared to be an appropriate rate of AP but prescribing patterns suggest a reactive rather than anticipatory approach, with some lack of continuity between inpatient and discharge prescriptions. More education and research are needed to support earlier identification of those in need of $\mathrm{AP}$ and smoother transitions between the inpatient and community setting.

\section{ADDRESSING PATIENTS' HYDRATION NEEDS AT THE END OF LIFE: A CROSS-SITE AUDIT}

Amy Hawkins, Beata LeBon. Frimley Park Hospital

\subsection{6/bmjspcare-2018-ASPabstracts.86}

Background Assessment of hydration needs including, if appropriate, a trial of clinically assisted hydration $(\mathrm{CAH})$ is a key recommendation of national guidelines for dying patients. ${ }^{1,2}$ There is insufficient evidence regarding the benefits and burdens of $\mathrm{CAH}$ at the end of $\operatorname{life}^{3,4,5}$ including inconclusive findings from a systematic review. ${ }^{6}$ Research is ongoing ${ }^{7}$ including a cluster RCT. $^{8}$ The audit aim was to assess compliance with national guidelines in three settings: the hospice inpatient unit (IPU), a district general hospital and patients known to the community palliative care team.

Methods Ten audit standards were derived from NICE and GMC guidelines. ${ }^{1,2}$ A target of $100 \%$ compliance with each standard was set. The audit comprised retrospective case note review of 60 patients (20 in each setting), identified as consecutive deaths of patients on an individualised end of life care plan from 1 st October 2016.

Results The frequency of documented mouth care and hydration assessment did not meet the audit standards in any setting. Most hospital inpatients had $\mathrm{CAH}$ in the last week of life $(90 \%)$, compared with $10 \%$ of IPU patients and none of the community cohort. Discussion regarding risks and benefits of $\mathrm{CAH}$ was not widely documented, with the lowest figures for community (5\%) and IPU patients (15\%). In all cases in which CAH was started, the documented frequency of reassessment was less than the twice daily recommendation in national guidelines. ${ }^{1}$

Discussion In part, some of the findings may reflect incomplete documentation. Since the audit, the following steps have been taken:

- Review of hydration needs assessment including changes to care plan documentation

- Meetings with district nursing teams

- Review of access to required equipment for administering $\mathrm{CAH}$

- Education sessions for staff

- Review of hospital mouth care guidelines

We plan to re-audit to assess compliance with the guidelines following implementation of these changes.

\section{MULTI ORGANISATIONAL AUDIT OF THE RECORD AND PROMPT FOR ADULT CARE IN THE LAST DAYS OF LIFE IN WIRRAL}

Catherine Hayle, Gursaran Purewal, Emma Longford, Fawad Ahmad, Richard Latten, CarlaJayne Lunt, Daniel Evans, Clare Brown, Penny Shephard, Jill Littlewood, Nicola D'Amelio. Wirral University Teaching Hospitals NHS Foundation Trust, Wirral Hospice St John's

\subsection{6/bmjspcare-2018-ASPabstracts.87}

Background The Wirral Multidisciplinary Record and Prompt for Adult Care in the Last Days of Life was developed in 2016 to support the delivery of excellent individualised care in last days of life in keeping with the Priorities for Care of the Dying Person and NICE guidance for Care of Dying Adults in the Last Days of Life. Following its introduction across Wirral University Teaching Hospitals NHS Foundation Trust and Wirral Hospice St John's, audits were undertaken across both settings assessing whether care in the last days of life was delivered and documented in line with national guidance.

Method A retrospective case note review was conducted using a standardised data collection tool. In the hospital setting, the audit sample from January-March 2017 included 30 cases where care was supported with the record of care, and 29 cases where it was not. In the hospice setting the audit sample from June-September 2016 included 34 patients whose care had been supported using this tool with comparison to a previous audit in 2015 assessing care in the last days of life.

Results The results across both organisations showed a considerable improvement in care delivered to patients in the last days of life, when the record of care was used, as evidenced by key findings in table 1 and 2 .

Conclusion The use of the record of care has led to meaningful improvements in the care documented for dying patients and those close to them across all domains. The tool has been embedded across both organisations, with the focus now on ongoing education, awareness-raising of the improvement seen when the tool is used, and developments of electronic templates to support its use. Roll-out across the community setting is planned during 2018, with the aim of assuring the same high quality care in any setting. 